\title{
Fingerprint image segmentation using modified fuzzy c-means algorithm
}

\author{
Jia-Yin Kang ${ }^{1}$, Cheng-Long Gong ${ }^{1}$, Wen-Juan Zhang ${ }^{2}$
}

${ }^{1}$ School of Electronics Engineering, Huaihai Institute of Technology, Lianyungang, China;

${ }^{2}$ School of Computer Engineering, Huaihai Institute of Technology, Lianyungang, China.

Email: jiayinkang@gmail.com

Received 16 July 2009; revised 31 August 2009; accepted 1 September 2009.

\begin{abstract}
Fingerprint segmentation is a crucial step in fingerprint recognition system, and determines the results of fingerprint analysis and recognition. This paper proposes an efficient approach for fingerprint segmentation based on modified fuzzy c-means (FCM). The proposed method is realized by modifying the objective function in the Szilagyi's algorithm via introducing histogram-based weight. Experimental results show that the proposed approach has an efficient performance while segmenting both original fingerprint image and fingerprint images corrupted by different type of noises.
\end{abstract}

Keywords: Fingerprint; Segmentation; Fuzzy C-means; Histogram; Robustness

\section{INTRODUCTION}

Fingerprint segmentation is an important issue in fingerprint recognition system. A fingerprint image usually has to be segmented to remove uninterested regions before some other steps such as enhancement and minutiae detection so that the image processing will consume less CPU time. A fingerprint image generally consists of different regions: non-ridge regions, high quality ridge regions, and low quality ridge regions. Fingerprint segmentation is usually to identify non-ridge regions and unrecoverable low quality ridge regions and exclude them as background [1]. Most segmentation methods are block-wised ones which divide the fingerprint image into un-overlapped blocks and decide on the type (background and foreground) of each block. Some other methods are pixel-wised ones which determine the type of each pixel. Fingerprint segmentation typically computes the feature (or feature vector) of each element, block or pixel, and then determines the element's type based on the feature (vector). The features used in fingerprint segmentation mainly include statistical features of pixel intensity, directional image and ridge projection signal et al.
Fuzzy c-means (FCM) clustering algorithm, an unsupervised clustering technique, has been widely used in image segmentation since it was proposed [2,3]. Compared with hard c-means algorithm [4], FCM is able to preserve more information from the original image. However, for one thing, it is noise-sensitive for not taking into account the spatial information [5]; for another, it is supposed that each feature date has the same contribution to classifying results [6]. To solve the first problem, recently, many researchers proposed the algorithms accounting for spatial information via modifying the objective function of standard FCM algorithm $[5,7,8]$. To solve the second problem, Li et al. [6] proposed a modified clustering algorithm via introducing feature weight of the data.

Generally, fingerprint image is gray level image and is inevitably corrupted by noise during acquisition. Consequently, data feature of the fingerprint is the pixel's gray value. From the gray level histogram of the fingerprint image, it is easily known that the occurrence frequencies of the different gray levels are usually different. Therefore, different gray level pixel has different contribution to clustering results.

In this paper, we propose a modified algorithm for noisy fingerprint image segmentation. The proposed approach is based on modified fuzzy c-means which is robust to noise. Our method achieves more desirable performance compared to standard FCM and Szilagyi modified FCM in [8].

The paper is organized as follows. In Subsection 2.1, standard FCM clustering algorithm is described. Subsection 2.2 presents the proposed modified FCM algorithm to segment the fingerprint. In Section 3, the experimental results are presented. Finally, Section 4 gives our conclusions and discussions.

\section{METHODOLOGY}

\subsection{Standard FCM Algorithm}

The FCM algorithm assigns pixels to each category by using fuzzy memberships. 
Let $X=\left\{x_{i}, i=1,2, \cdots, N \mid x_{i} \in \mathbb{R}^{d}\right\}$ denotes an image with $N$ pixels to be partitioned into $c$ classes, where $x_{i}$ represents features data. The algorithm is an iterative optimization that minimizes the objective function defined as follows [3]:

$$
J_{m}=\sum_{k=1}^{c} \sum_{i=1}^{N} u_{k i}^{m}\left\|x_{i}-v_{k}\right\|^{2}
$$

with the following constraints:

$$
\left\{u_{k i} \in[0,1] \mid \sum_{k=1}^{c} u_{k i}=1, \forall i, 0<\sum_{i=1}^{N} u_{k i}<N, \forall k\right\}
$$

where $u_{k i}$ represents the membership of pixel $x_{i}$ in the $k^{\text {th }}$ cluster, $v_{k}$ is the $k^{\text {th }}$ class center; $\|\bullet\|$ denotes the Euclidean distance, $m>1$ is a weighting exponent on each fuzzy membership. The parameter $m$ controls the fuzziness of the resulting partition. The membership functions and cluster centers are updated by the following expressions:

$$
u_{k i}=\frac{1}{\sum_{l=1}^{c}\left(\frac{\left\|x_{i}-v_{k}\right\|}{\left\|x_{i}-v_{l}\right\|}\right)^{2 /(m-1)}}
$$

and

$$
v_{k}=\frac{\sum_{i=1}^{N} u_{k i}^{m} x_{i}}{\sum_{i=1}^{N} u_{k i}^{m}}
$$

In implementation, matrix $V$ is randomly initialized, and then $U$ and $V$ are updated through an iterative process using Eq.s 3 and 4 respectively.

\subsection{Modified FCM Algorithm}

Szilagyi et al.proposed a fast FCM clustering algorithm, EnFCM [8], which is used for gray level image segmentation. The algorithm accounts for pixel spatial information. Before the algorithm implementation, a linearlyweighted sum image $\xi$, composed by original image and local neighboring average of each pixel in original image, was calculated as follows:

$$
\xi_{i}=\frac{1}{1+\alpha}\left(x_{i}+\frac{\alpha}{N_{R}} \sum_{j \in N_{i}} x_{j}\right)
$$

where $\xi_{i}$ is the gray value of the $i^{\text {th }}$ pixel in the image $\xi$. $N_{i}$ stands for the set of neighbors falling into a local window around $x_{i}$, and $N_{R}$ is its cardinality. The parameter $a$ in the second term controls the effect of the penalty. In essence, the addition of the second term in Eq.5, equivalently, formulates a spatial constraint and aims at keeping continuity on neighboring pixel values around $x_{i}$. Accordingly, the modified objective function was described as follows:

$$
J_{s}=\sum_{k=1}^{c} \sum_{l=1}^{q} \gamma_{l} u_{k l}^{m}\left\|\xi_{l}-v_{k}\right\|^{2}
$$

where $\xi=\left\{\xi_{l}, l=1,2, \ldots, q\right\}$ is the data set rearranging from he image $\xi$ defined in Eq.5 according to gray level. $V=\left\{v_{k}\right\}(k=1,2, \cdots, c)$ represents the prototype of the $k^{\text {th }}$ cluster, $U=\left\{u_{k l}\right\}(k=1,2, \cdots, c, l=1,2, \cdots, q)$ represents the fuzzy membership of gray value $l$ with respect to cluster $k \cdot q$ denotes the number of the gray level of the given image which is generally much smaller than $N . \gamma_{l}$ is the number of the pixels having the gray value equal to $l$, where $l=1,2, \cdots, q$. Naturally, $\sum_{l=1}^{q} \gamma_{l}=N$.

Similar to the standard FCM algorithm, under the constraints that $\sum_{k=1}^{c} u_{k l}=1$ for any $l$, minimize $J_{s}$ defined in Eq.6. Specifically, taking the first derivatives of $J_{s}$ with respect to $u_{k l}$ and $v_{k}$, and zeroing them, respectively, two necessary but not sufficient conditions for $J_{s}$ will be obtained as follows:

$$
\begin{gathered}
u_{k l}=\frac{\left(\xi_{l}-v_{k}\right)^{-2 /(m-1)}}{\sum_{r=1}^{c}\left(\xi_{l}-v_{r}\right)^{-2 /(m-1)}} \\
v_{k}=\frac{\sum_{l=1}^{q} \gamma_{l} u_{k l}^{m} \xi_{l}}{\sum_{l=1}^{q} \gamma_{l} u_{k l}^{m}}
\end{gathered}
$$

Obviously, in Eq.6, gray level was viewed as the classified data. Hence, the number of classified data only depends on gray level, and doesn't enlarge with the increasing of image size. However, Eq.6 doesn't take different gray level which has different influence on classifying results into consideration, i.e., Eq.6 considers that every gray level has the same contribution to the classifying results. Actually, according to the gray level histogram of the fingerprint image, it is clear that the occurrence frequencies of different gray level are different. Therefore, different gray level has different contribution to clustering results. Based on above analysis, we modified the objective function in Eq.6 as follows:

$$
J_{s}=\sum_{k=1}^{c} \sum_{l=1}^{q} w_{l} \gamma_{l} u_{k l}^{m}\left\|\xi_{l}-v_{k}\right\|^{2}
$$

where $w_{l}$ is the weighting coefficient of $\zeta_{l}(l=1,2, \cdots, q)$, and can be computed via histogram as follows:

$$
w_{l}=\frac{\gamma_{l}}{N}, \quad l=0,1, \cdots, q
$$

where $q$ denotes the number of the gray level of the given image. $\gamma_{l}$ is the number of the pixels having the 
gray value equal to $l$, where $l=1,2, \cdots, q$. Naturally, $\sum_{l=1}^{q} \gamma_{l}=N, \quad \sum_{l=1}^{q} w_{l}=1$, i.e., $w_{l}(l=1,2, \cdots, q) \quad$ can be viewed as the occurrence probability of each gray level. Hence, from Eq.10, it is known that the weighting coefficient of each gray level can be given by the normalized image histogram.

Similarly, under the constraints that $\sum_{k=1}^{c} u_{k l}=1$ for any $l$, minimize $J_{s}$ defined in Eq.9. Specifically, taking the first derivatives of $J_{s}$ with respect to $u_{k l}$ and $v_{k}$, and zeroing them, respectively, two necessary but not sufficient conditions for $J_{s}$ will be obtained as follows:

$$
\begin{gathered}
u_{k l}=\frac{\left(\xi_{l}-v_{k}\right)^{-2 /(m-1)}}{\sum_{r=1}^{c}\left(\xi_{l}-v_{r}\right)^{-2 /(m-1)}} \\
v_{k}=\frac{\sum_{l=1}^{q} w_{l} \gamma_{l} u_{k l}^{m} \xi_{l}}{\sum_{l=1}^{q} w_{l} \gamma_{l} u_{k l}^{m}}
\end{gathered}
$$

From Eq.12, it is known that the function of weighting coefficient $w_{l}$ lies in adjusting the clustering center. Eq.9 will degenerated to Eq.6 while $w_{l}=1 / q$.

The modified FCM algorithm (spatially weighting FCM clustering algorithm, called SWFCM) can be summarized as follows:

Step 1: Fix $m>1$ and $2 \leq c \leq N-1$; then select initial class prototypes $v_{k}(k=1,2, \cdots, c)$; set $\varepsilon>0$ to a very small value.

Step 2: Compute the new image $\zeta$ in terms of Eq.5 in advance.

Repeat:

Step 3: Compute/modify $\mu_{k l}$ with $v_{k}$ by Eq.s 11 and 12 .

Step 4: Update $v_{k}$ with the modified $\mu_{k l}$ by Eq. 12 . Until $\left(\left|V_{\text {new }}-V_{\text {old }}\right|<\varepsilon\right)$

\section{RESULTS AND DISCUSSIONS}

In the following experiments, we first execute the three segmentation algorithms, FCM, EnFCM and SWFCM on an original fingerprint image in Subsection 3.1. Then perform the three segmentation algorithms on noisy fingerprint images corrupted, respectively, by Gaussian noise and salt and pepper noise to investigate the robustness of the algorithms in Subsection 3.2. Finally, the Subsection 3.3 gives the corresponding quantitative comparisons for the segmenting results presented in Subsection 3.1 and 3.2. In all the following experiments, we set the parameters $c=2, m=2, a=5, \varepsilon=10^{-5}$.

\subsection{Results on Original Fingerprint Image}

To compare the segmenting performances of the above three algorithms, FCM, EnFCM and SWFCM, we apply these algorithms to an original test fingerprint image as shown in Figure 1(a) with the size of $300 \times 300$ pixels, and the corresponding segmenting results are, respectively, displayed in Figures 1(b,c,d).

As shown in Figure 1, it is clear that our proposed algorithm performs more visually significant than other two methods do. More detailed quantified comparison according to execution time and iteration step are given in the next subsection.

\subsection{Results on Fingerprint Images Corrupted by Noises}

To examine the above three algorithms' robustness to noise, we apply the three algorithms on fingerprint images corrupted by noises. Figure 2(a) is the original image with no noise and Figures 2(b) and 2(c) are the same images corrupted, respectively, by the Gaussian noise ( $\mu=0, \sigma=0.05)$ and the salt and pepper noise with noisy density $d=0.02$. The segmenting results on Figures 2(a) and 2(b) are shown in Figures 3 and 4, respectively.

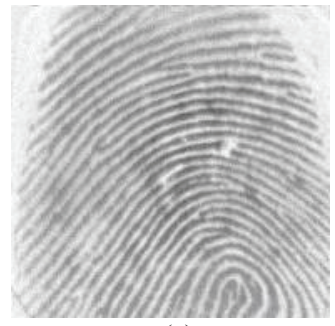

(a)

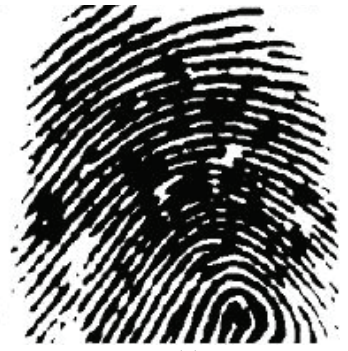

(c)

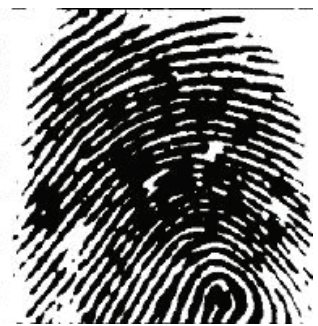

(b)

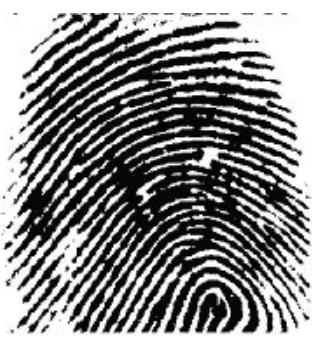

(d)
Figure 1. Fingerprint image segmentation. (a) Original fingerprint image; (b) Fingerprint segmentation result using FCM; (c) Fingerprint segmentation result using EnFCM; (d) Fingerprint segmentation result using SWFCM.

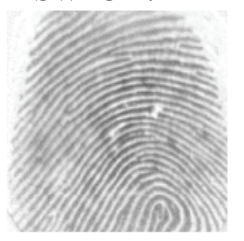

(a)

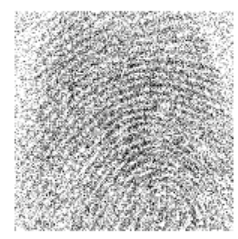

(b)

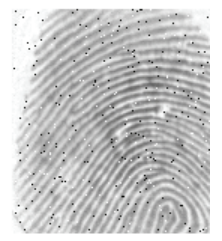

(c)
Figure 2. Fingerprint image. (a) Original image; (b) The image corrupted by Gaussian noise; (c) The image corrupted by salt and pepper noise. 

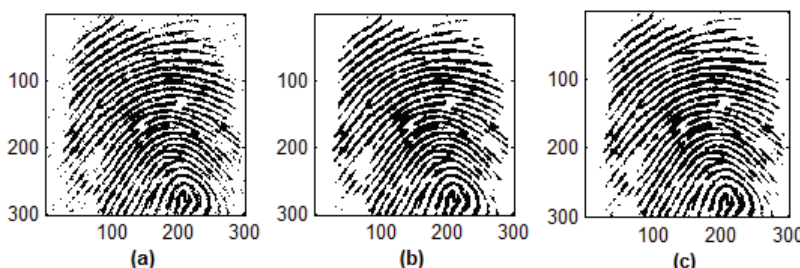

Figure 3. Segmenting results on fingerprint image corrupted by Gaussian noise. (a) Fingerprint segmentation result using FCM (b) Fingerprint segmentation result using EnFCM (c) Fingerprint segmentation result using SWFCM.

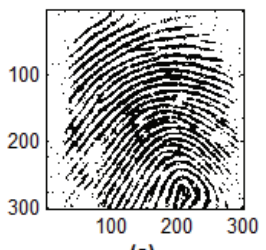

(a)

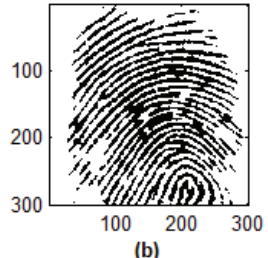

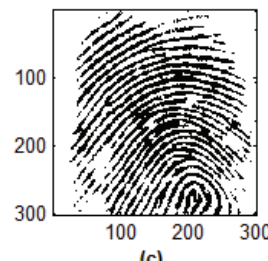

Figure 4. Segmenting results on fingerprint image corrupted by salt and pepper noise. (a) Fingerprint segmentation result using FCM; (b) Fingerprint segmentation result using EnFCM; (c) Fingerprint segmentation result using SWFCM.

Both from Figures 3 and 4, We can visually see that FCM is influenced by the Gaussian noise and the salt and pepper noise to different extents, respectively, which indicate that FCM algorithm lacks enough robustness to both the Gaussian noise and the salt and pepper noise, while EnFCM and SWFCM can basically eliminate the effect of the noises. Although the segmenting results using EnFCM and SWFCM are visually almost same, more detailed quantified comparison according to execution time, iteration step and signal-to-noise ratio (SNR) are needed to further investigate in the next subsection.

\subsection{Quantitative Segmenting Results Comparisons}

We tabulate quantitative segmenting comparisons in Tables 1,2,3 of the above three algorithms for Figures 1,3,4, respectively.

From Tables $1,2,3$, we can obviously see that the iteration step of SWFCM is less than that of FCM and EnFCM. Furthermore, the execution time (CPU: $2 \mathrm{GHz}$, Memory: $1 \mathrm{GHz}$, Operating system: windows XP, Soft ware: Matlab 7.0) of SWFCM is reduced compared with FCM and EnFCM due to both the less iterations and only dependence on the number of the gray-level $q(256)$ rather than the image size itself $N(300 \times 300)$.

Table 1. Comparison scores of three methods corresponding to Figure 1.

\begin{tabular}{ccc}
\hline Algorithm & $\mathrm{T}$ & $\mathrm{N}$ \\
\hline FCM & 5.123 & 35 \\
EnFCM & 3.841 & 28 \\
SWFCM & 3.077 & 23 \\
\hline
\end{tabular}

Table 2. Comparison scores of three methods corresponding to Figure 3.

\begin{tabular}{lccc}
\hline Algorithm & $\mathrm{T}$ & $\mathrm{N}$ & $\mathrm{SNR}$ \\
\hline FCM & 3.198 & 17 & 28.075 \\
EnFCM & 2.573 & 13 & 28.591 \\
SWFCM & 1.967 & 11 & 31.308 \\
\hline
\end{tabular}

Table 3. Comparison scores of three methods corresponding to Figure 4.

\begin{tabular}{lccc}
\hline Algorithm & $\mathrm{T}$ & $\mathrm{N}$ & $\mathrm{SNR}$ \\
\hline FCM & 3.130 & 14 & 22.157 \\
EnFCM & 2.217 & 8 & 24.972 \\
SWFCM & 1.048 & 4 & 27.872 \\
\hline
\end{tabular}

Note: In the above three tables, $\mathrm{T}$ stands for execution time with the unit of second; $\mathrm{N}$ stands for iteration step.

From Tables 2 and 3, we can also see that the SNR of SWFCM is less than that of FCM and EnFCM. From this results, it can be concluded that the newly-proposed algorithm give rise to better denoising performance than both FCM and EnFCM algorithms.

\section{CONCLUSIONS}

In this paper, an automatic modified FCM clustering algorithm for fingerprint segmentation was proposed. The proposed algorithm is realized by modifying the objective function in the Szilagyi's algorithm via introducing the gray histogram-based weighting. Experimental results show that proposed method can dramatically speed up FCM, and can achieve better denoising performance compared with EnFCM. Therefore, the proposed approach will be promising in real fingerprint recognition system, in which the fingerprint images are contaminated by noises heavily.

\section{ACKNOWLEDGMENTS}

The authors would like to address appreciations to anonymous reviewers for their valuable comments and suggestions to improve the presentation of this paper. This work was supported by the Jiangsu Postdoctoral Science Foundation (Grant No. 0901077C), the HHIT Science Foundation (Grant No. Z2008035) and Postdoctoral Science Foundation of China (Grant No. 20090451167).

\section{REFERENCES}

[1] J. P. Yin, E. Zhu and X. J. Yang. (2007) Two steps for fingerprint segmentation. Image and Vision Computing, 25, 1391-1403.

[2] D. Zhang and Y. Wang. (2006) Medical image segmentation based on FCM clustering and rough set. Chinese Journal of Scientific Instrument, 27, 1683-1687.

[3] W. J. Chen, M. L. Giger and U. Bick. (2006) A fuzzy c-means (FCM)-based approach for computerized segmentation of breast lesions in dynamic contrast-enhanced MR images. Academic Radiology, 13, 63-72.

[4] J. M. Gorriz, J. Ramirez and E. W. Lang. (2006) Hard c-means clustering for voice activity detection. Speech Communication, 48, 1638-1649.

[5] K. S. Chuang, H. L. Tzeng and S. W. Chen. (2006) Fuzzy 
c-means clustering with spatial information for image segmentation. Computerized Medical Imaging and Graphics, 30, 9-15.

[6] J. Li, X. B. Gao and L. C. Jiao, (2006) A new feature weighted fuzzy clustering algorithm, Acta Electronica Sinica, 34, 89-92.

[7] S. C. Chen and D. Q. Zhang. (2004) Robust image seg- mentation using FCM with spatial constraints based on new kernel-induced distance measure. IEEE Trans. Systems Man Cybernet, B, 34, 1907-1916.

[8] L. Szilagyi, Z. Benyo and S. M. Szilagyii. (2003) MR brain image segmentation using an enhanced fuzzy cmeans algorithm. 25th Annual International Conference of IEEE EMBS, 17-21. 